\title{
INTRODUCTION TO 4D CAUSAL DYNAMICAL TRIANGULATIONS*
}

\author{
ANDRZEJ GÖRLICH \\ The Marian Smoluchowski Institute of Physics, Jagiellonian University \\ Reymonta 4, 30-059 Kraków, Poland
}

(Received November 29, 2013)

\begin{abstract}
The model of Causal Dynamical Triangulations is a nonperturbative and background independent approach to quantum theory of gravity. In this paper, we give introduction to the four dimensional framework of Causal Dynamical Triangulations and present recent results. We describe the phase structure of the model and show how a macroscopic four dimensional de Sitter universe emerges dynamically from the full gravitational path integral. We advocate that the effective action describing scale factor fluctuations reconstructed from Monte Carlo data agrees with the minisuperspace model.
\end{abstract}

DOI:10.5506/APhysPolB.44.2559

PACS numbers: 98.80.Qc, 04.60.Gw, 04.60.Nc

\section{Introduction}

The model of Causal Dynamical Triangulations (CDT) is based on the formalism of path integrals and lattice regularization. Its objective is to quantize a theory of gravitation. The model was presented some years ago by Ambjørn, Jurkiewicz and Loll in [1-3]. In the path integral formulation of quantum gravity, the trajectories are represented by four-dimensional geometries of spacetime. CDT defines the way the path integral should be calculated and specifies the class of spacetime histories which should contribute to the path integral. A discretization is introduced, but only as a regularization intended to be eliminated in the continuum limit. Discreteness is not claimed to be a property of the universe.

* Presented at the LIII Cracow School of Theoretical Physics "Conformal Symmetry and Perspectives in Quantum and Mathematical Gravity", Zakopane, Poland, June 28-July 7, 2013. 
According to General Relativity, the classical theory of gravitation, the gravity is encoded in spacetime geometry. The considered degree of freedom is the metric field $g_{\mu \nu}(x)$ and nonzero curvature is interpreted as a gravitational field. The construction of the quantum theory starts from the classical Einstein-Hilbert action

$$
S_{\mathrm{EH}}\left[g_{\mu \nu}\right]=\frac{1}{16 \pi G} \int_{\mathcal{M}} d^{4} x \sqrt{-\operatorname{det} g}(R-2 \Lambda),
$$

where $G$ and $\Lambda$ are respectively the Newton's gravitational constant and the cosmological constant, $\mathcal{M}$ is the spacetime manifold equipped with a pseudoRiemannian metric $g_{\mu \nu}$ with Minkowskian signature $\{-,+,+,+\}$ and $R$ denotes the associated Ricci scalar curvature $[4,5]$. We used the natural Planck units $c=\hbar=1$ and assumed the topology of $\mathcal{M}$ to be $S^{1} \times S^{3}$.

In order to quantize the theory of gravitation, we apply the Feynman's path integral formalism. The path integral or partition function of quantum gravity is defined as a formal integral over all spacetime geometries

$$
Z=\int \mathcal{D}_{\mathcal{M}}[g] e^{i S_{\mathrm{EH}}[g]} .
$$

A spacetime geometry $[g]$, also called history, is defined as an equivalence class of spacetime metrics $g$ with respect to the diffeomorphism group on $\mathcal{M}$.

The Causal Dynamical Triangulations model regularizes the formal partition function (2) via discretization. The path integral is replaced by a sum over a discrete set $\mathbb{T}$ of all causal triangulations $\mathcal{T}$, thus it gains a meaningful definition. The building blocks of four dimensional CDT are four-simplices. A four-simplex is a generalization of a triangle to four dimensions, it is

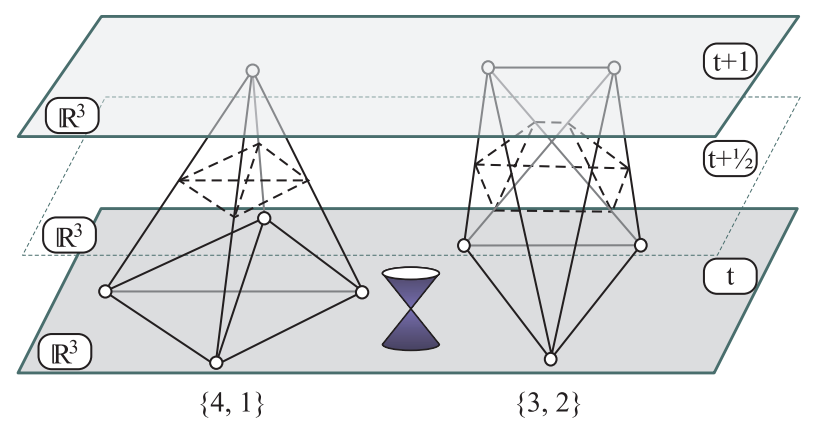

Fig. 1. A sketch of four-simplices, the fundamental building blocks of fourdimensional Causal Dynamical Triangulations. The simplices join two successive slices $t$ and $t+1$, and are grouped into two types: $\{4,1\}$ and $\{3,2\}$. The simplices are equipped with the flat Minkowski metric imposing the light-cone structure. 
composed of five vertices connected to each other. It is a subset of a fourdimensional Minkowski spacetime and the induced metric inside a simplex is flat. Figure 1 presents a sketch of four-simplices together with a lightcone. A four-dimensional simplicial manifold is built from four-simplices glued pairwise along tetrahedral faces so that topological conditions are preserved. The curvature is localized on triangles and, in general, such complex cannot be embedded in $\mathbb{R}^{4}$.

\subsection{Causality}

Opposite to Euclidean Dynamical Triangulations (EDT) [8], Causal Dynamical Triangulations assume global proper-time foliation. In the Euclidean version, configurations are dominated by creation of baby universes and causal singularities. In order to prevent such behavior, CDT implements the causality condition. Also, the original Lorentzian signature of spacetime suggests that only causal geometries should contribute to the gravitational path integral $[6,7]$. The leaves of the time foliation, called slices, are spatial three-dimensional Cauchy surfaces $\Sigma$ with fixed topology. Figure 2 illustrates a triangulation with imposed foliation which violates the causality condition. We chose the topology of slices to be a three sphere $\Sigma=S^{3}$ and establish periodic boundary conditions in the time direction. Therefore, the spacetime topology is $\mathcal{M}=S^{1} \times S^{3}$.

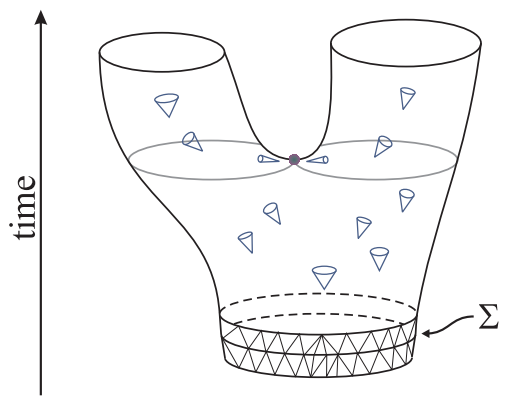

Fig. 2. A visualization of a two-dimensional triangulation with a light-cone structure and a branching point marked. In Causal Dynamical Triangulations spatial slices are not allowed to split, which prevents singularities of the time arrow.

On piecewise linear manifolds, the spatial slices $\mathcal{T}^{(3)}(i)$ are enumerated by a discrete time coordinate $i$. Each slice is a triangulation of $S^{3}$ made up of equilateral tetrahedra with a side length $a_{\mathrm{s}}>0$. The induced metric of spatial tetrahedra has a Euclidean signature. Each vertex of the triangulation is assigned a discrete time coordinate $i$ which corresponds to a slice it lies in. A layer between two successive slices $\mathcal{T}^{(3)}(i)$ and $\mathcal{T}^{(3)}(i+1)$ is filled 
with four-simplices so that it forms of a four-dimensional slab with a topology of $[0,1] \times S^{3}$. A series of slabs glued one after another builds the whole simplicial complex and preserves the causal structure. The triangulation of the later slice wholly lies in the future of the earlier one.

Due to the foliation, there are two kinds of simplices. Simplices of the first type are called $\{4,1\}$ and have four vertices in one spatial slice, while the fifth vertex lies in the previous or next slice. Simplices of the second type, called $\{3,2\}$, have three vertices in one spatial slice and two in the previous or next slice. Figure 1 illustrates four-simplices of type $\{4,1\}$ and $\{3,2\}$ connecting slices $\mathcal{T}^{(3)}(t)$ and $\mathcal{T}^{(3)}(t+1)$. There are also two kinds of links. The space-like links connect two vertices in the same slice and have length $a_{\mathrm{s}}>0$. The time-like links connect two vertices in adjacent slices and have length $a_{\mathrm{t}}$. In Causal Dynamical Triangulations, the lengths $a_{\mathrm{s}}$ and $a_{\mathrm{t}}$ are constant but not necessarily equal. Let us introduce the asymmetry factor $\alpha$ such that $a_{\mathrm{t}}^{2}=\alpha \times a_{\mathrm{s}}^{2}$. In the Lorentzian case, $\alpha$ is negative. The volumes and angles of simplices are fully determined by $a_{\mathrm{s}}$ and $a_{\mathrm{t}}$. They differ for the two types of simplices. Since no coordinates are introduced, the CDT model is manifestly diffeomorphism-invariant. Such a formulation involves only geometric invariants like lengths and angles.

\subsection{Lattice regularization and the Regge action}

The Einstein-Hilbert action (1) has a natural realization on piecewise linear geometries. Let us denote the number of simplices of type $\{4,1\}$ by $N_{41}$, and the number of simplices of type $\{3,2\}$ by $N_{32}$. The total number of simplices is then given by $N_{4}=N_{41}+N_{32}$. The total physical four-volume $\int_{\mathcal{T}} d^{4} x \sqrt{|\operatorname{det} g|}$ can be expressed by a linear combination of $N_{41}$ and $N_{32}$. Similarly, the global curvature $\int_{\mathcal{T}} d^{4} x \sqrt{|\operatorname{det} g|} R$ can be expressed using the angle deficits which are localized at triangles, and is a linear function of $N_{32}, N_{41}$ and the number of vertices $N_{0}$. The Einstein-Hilbert action (1) calculated for a causal triangulation $\mathcal{T}$ is called the Regge action and can be written in a very simple form

$$
S[\mathcal{T}] \equiv-K_{0} N_{0}[\mathcal{T}]+K_{4} N_{4}[\mathcal{T}]+\Delta\left(N_{41}[\mathcal{T}]-6 N_{0}[\mathcal{T}]\right)
$$

where $K_{0}, K_{4}$ and $\Delta$ are bare coupling constants. They are nonlinear functions of $G$ and $\Lambda$ appearing in the continuous (1) action, and the asymmetry factor $\alpha$ [3]. As the bare coupling constant $K_{4}$ is conjugate to $N_{4}$, it controls the total volume and can be considered as a cosmological constant. $K_{0}$ is related to the inverse of the gravitational coupling constant $G$ and $\Delta$ is related to the asymmetry factor $\alpha$. $\Delta$ will play an important role as it will allow observing new geometric phases. 
Causal Dynamical Triangulations provide a lattice regularization of histories appearing in the formal gravitational path integral (2). The discretization procedure replaces the integral with a sum over the set of all causal triangulations $\mathbb{T}$ weighted by the Regge action (3), providing a meaningful definition of the partition function

$$
Z \equiv \sum_{\mathcal{T} \in \mathbb{T}} e^{i S[\mathcal{T}]}
$$

\subsection{The Wick rotation}

In contrast to the two dimensional case, lack of analytical tools in four dimensions forces us to use numerical methods to gain the nonperturbative information about fluctuating geometries. The number of configurations is finite for finite size triangulations. Nevertheless, this number grows exponentially with the number of simplices $N_{4}$ and the oscillatory nature of the integrand (4) precludes usage of numerical techniques. We may bypass this problem by applying a trick called Wick rotation. Roughly, it is based on the analytic continuation of the time coordinate to imaginary values. Hence, the spacetime signature changes from Lorentzian to Euclidean and complex amplitudes are substituted by real probabilities $e^{i S^{\mathrm{Lor}}} \rightarrow e^{-S^{\mathrm{Euc}}}$. Because of the global proper-time foliation, we have a distinction between time-like and space-like links, and the Wick rotation is well defined. It can be simply implemented by analytic continuation of the lengths of all time-like edges, $a_{\mathrm{t}} \rightarrow i a_{\mathrm{t}}$

$$
a_{\mathrm{t}}^{2}=\alpha a_{\mathrm{s}}^{2}, \quad \alpha>0 .
$$

The Regge action rotated to the Euclidean sector, after the redefinition $S^{\text {Euc }}=-i S^{\text {Lor }}$, has exactly the same simple form as its original Lorentzian version (3). An exact derivation of the Wick rotated Regge action can be found in [3].

As a consequence of the lattice regularization procedure and the Wick rotation, the partition function (2) is finally written as a real sum over the set of all causal triangulations $\mathbb{T}$

$$
Z=\sum_{\mathcal{T} \in \mathbb{T}} e^{-S[\mathcal{T}]} .
$$

Both the Euclidean Regge action $S[\mathcal{T}]$ as well as the partition function $Z$ depend on bare coupling constants $K_{0}, K_{4}$ and $\Delta$.

\subsection{The numerical setup}

The formal gravitational path integral (2), after Wick rotation, defines the quantum expectation value 


$$
\langle\mathcal{O}[g]\rangle \equiv \frac{1}{Z} \int \mathcal{D}[g] e^{-S_{\mathrm{EH}}[g]},
$$

where $\mathcal{O}[g]$ denotes some observable defined on a geometry $[g]$. Cognately, the probability distribution $P[\mathcal{T}]$ associated with the partition function (5), defines its discrete counterpart

$$
\langle\mathcal{O}[\mathcal{T}]\rangle \equiv \sum_{\mathcal{T} \in \mathbb{T}} \mathcal{O}[\mathcal{T}] P[\mathcal{T}], \quad P[\mathcal{T}] \equiv \frac{1}{Z} e^{-S[\mathcal{T}]}
$$

Here, $\mathcal{O}[\mathcal{T}]$ denotes an observable measured on triangulation $\mathcal{T}$.

The CDT framework, strictly based on lattice regularization, enables application of effective Monte Carlo techniques. The partition function (5) defines a statistical mechanical problem which is free of oscillations. It is the starting point for computer simulations which further allow us to measure expectation values defined by (7) in an approximate manner and gain physically relevant information. Numerical simulations consist in generating a sequence of spacetime geometries, being causal triangulations $\mathcal{T}$, according to the probability distribution (7). Given a sufficiently large set of $K$ statistically independent Monte Carlo configurations $\left\{\mathcal{T}^{(k)}, k=1 \ldots K\right\}$, we approximate the average (7)

$$
\langle\mathcal{O}[\mathcal{T}]\rangle \approx \frac{1}{K} \sum_{k=1}^{K} \mathcal{O}\left[\mathcal{T}^{(k)}\right] .
$$

The probability distribution $P[\mathcal{T}]$ is not evident in the above equation, because by construction the output configurations of the Monte Carlo algorithm are weighted by the Regge action.

\section{The macroscopic universe}

An example of the simplest observable providing information about the universe geometry is the spatial three-volume $n_{i}$. It is defined as a number of tetrahedra building a spatial slice $\mathcal{T}^{(3)}(i)$, where $i=1 \ldots T$. It sums up to the total volume $N=N_{41}=\sum_{i=1}^{T} n_{i}$ (up to a constant factor $\frac{1}{2}$ ).

An individual spacetime history contributing to the partition function is not an observable, precisely in the same way as a trajectory of a particle in the quantum-mechanical path integral is not an observable. However, it is perfectly legitimate to talk about the expectation value $\left\langle n_{i}\right\rangle$ as well as about the fluctuations around the mean.

Hereinafter, let us restrict our considerations to the spatial volume $n_{i}$. Hence, we reduce the problem to one-dimensional quantum mechanics. We should keep in mind that many three-dimensional triangulations of spatial slices might have the same spatial volume $n$. 


\subsection{The phase diagram}

The Regge action (3) has three bare coupling constants, namely the inverse of the gravitational constant $K_{0}$, the cosmological constant $K_{4}$ and the asymmetry constant $\Delta$. For technical reasons, it is convenient to keep the total four-volume $N_{4}$ fluctuating around some prescribed value during Monte Carlo simulations. The cosmological constant $K_{4}$ acts as Lagrange multiplier and needs to be tuned to its critical value in order to suppress the contribution to the partition function coming from extremely large configurations. Because the number of configurations grows exponentially with the size, a value of $K_{4}$ below the critical value would make the partition function ill-defined. Effectively, $K_{4}$ does not appear as a coupling constant.

We observe three qualitatively different behaviors of a typical configuration depending on the values of the two remaining bare coupling constants $K_{0}$ and $\Delta$. In [2] it was shown that the model consists of three phases, which were labeled $A, B$ and $C$. A detailed phase diagram obtained due to largescale computer simulations was described in [9]. The phase diagram, based on Monte Carlo measurements, is presented in Fig. 3. The solid lines denote observed phase transition points for configurations of size 80000 simplices, while the dashed lines represent an interpolation.

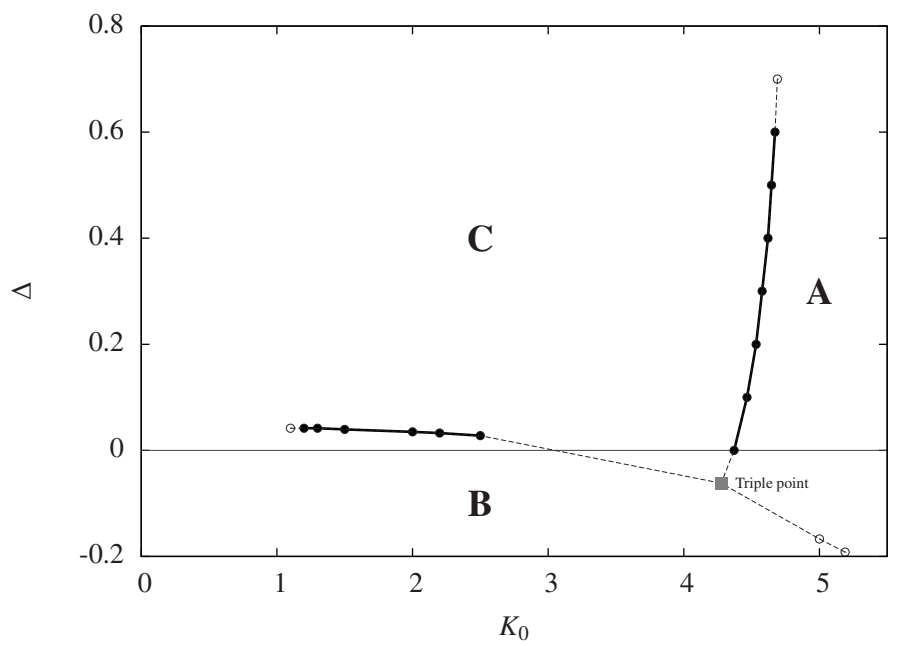

Fig. 3. A sketch of the phase diagram. The phases correspond to regions on the bare coupling constant $K_{0}-\Delta$ plane. We observe three phases: a crumpled phase $A$, a branched polymer phase $B$ and the most interesting a genuinely four-dimensional de Sitter phase $C$. 
Figure 4 displays volume profiles $n_{i}$ of typical configurations of the three phases:

- Phase A. For large values of $K_{0}$ (cf. Fig. 3), the universe disintegrates into uncorrelated irregular sequences of maxima and minima with time extent of few steps. When looking along the time direction, we observe a number of universes oscillating in the time direction which are connected by necks of negligible sizes.

- Phase B. For small values of $\Delta$, nearly all simplices collapse into one spatial slice. Although this slice has a topology of a three-sphere $S^{3}$, the measured Hausdorff dimension is very high, if not infinite.

- Phase C. For larger values of $\Delta$, a typical configuration is bell-shaped and behaves like a well defined four-dimensional manifold. The measurements of the Hausdorff dimensions confirm that at large scales the universe is genuinely four-dimensional [2].

Only in the latter phase, the configurations have a finite time and spatial extents, which scale as expected for a four-dimensional object. Hence, only phase $C$ is regarded physical. The averaged profile $\left\langle n_{i}\right\rangle$ corresponds to Euclidean de Sitter space $S^{4}$ and thus this phase is also called the de Sitter phase.
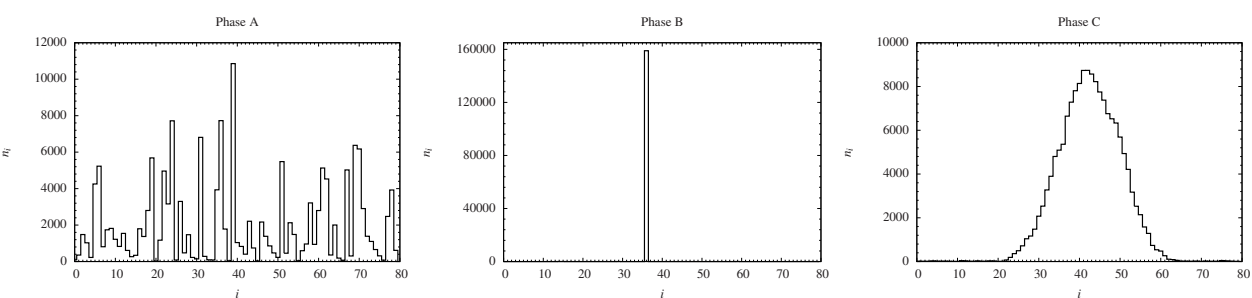

Fig. 4. Snapshot of a spatial volume $n_{i}$ for a typical configuration of phases $A, B$ and $C$. A typical configuration in phase $C$ is bell-shaped with well-defined spatial and time extent.

\subsection{The de Sitter universe}

In phase $C$, the time translational symmetry is spontaneously broken and the distribution $n_{i}$ is bell-shaped. Let us focus on point $K_{0}=2.2, \Delta=0.6$ of the phase diagram which is firmly placed in phase $C$. A snapshot of a volume profile $n_{i}$ of a typical configuration with the total volume $N=160000$ simplices and time-period $T=80$ is shown in Fig. 5. For this value of $N$, the universe does not extend over the entire time-axis, but is localized in 
a much shorter range. The Regge action, as derived from the EinsteinHilber action, is time translationally invariant. For time-periodic boundary conditions, a direct average $\left\langle n_{i}\right\rangle$ would give a constant function of time $i$, not capturing the shape of individual configurations. Therefore, to obtain a meaningful expectation value of $n_{i}$, we fix the position of the center of mass before performing the average over triangulations (8) [10].

Figure 5 shows the measured $\left\langle n_{i}\right\rangle$ (black thick line). The boxes visible on the plot indicate the amplitude of quantum fluctuations of $n_{i}$, their height is equal to standard deviation $\sigma_{i}=\sqrt{\left\langle n_{i}^{2}\right\rangle-\left\langle n_{i}\right\rangle^{2}}$. The average volume $\left\langle n_{i}\right\rangle$ is with high accuracy given by formula

$$
\left\langle n_{i}\right\rangle=H \cos ^{3}(i / W) .
$$

Here, $W$ is the time extent of the universe and $H$ is the maximal spatial volume. The function (9) with fitted $H$ and $W$ is plotted in Fig. 5 with a dashed line, but it is indistinguishable from the measured curve. The formula (9) describes the background geometry. It was not put by hand but emerged dynamically. Moreover, it corresponds to the maximally symmetric solution of classical Einstein equations with a positive cosmological constant [11, 12], i.e. Euclidean de Sitter space - geometry of a four-sphere $S^{4}$. Although by construction the topology of the spacetime is enforced to be $\mathcal{M}=S^{1} \times S^{3}$, in the large $N$ limit the stalk can be neglected and the topology transmutes into $S^{4}$.

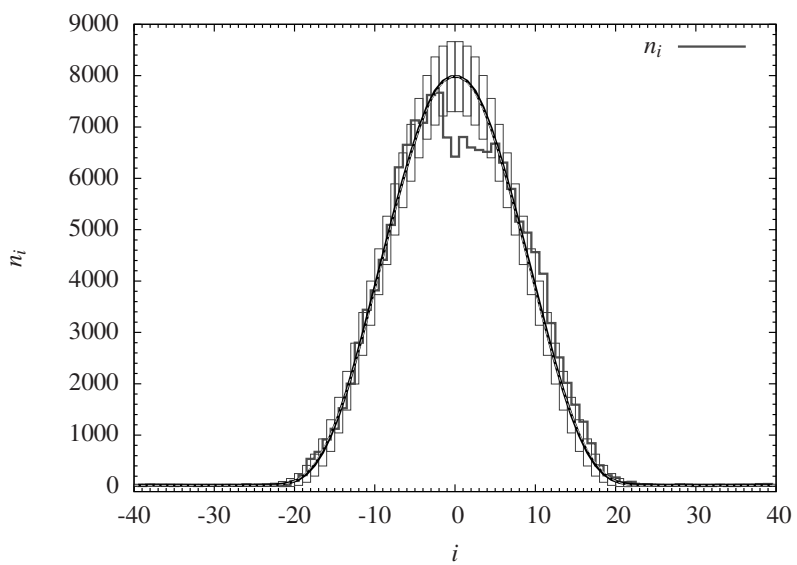

Fig. 5. Spatial volume $n_{i}$ of a randomly chosen typical configuration (gray line) and the background geometry $\left\langle n_{i}\right\rangle$ (black line): Monte Carlo measurements for fixed $N_{41}=160000, K_{0}=2.2, \Delta=0.6$. The best fit (9) yield indistinguishable curves at given plot resolution. The bars height indicate the average size of quantum fluctuations. 


\subsection{The minisuperspace model}

The solution (9) $\left\langle n_{i}\right\rangle=H \cos ^{3}(i / W)$ comes as a classical solution of the minisuperspace model. This quantum cosmological model was developed by Hartle and Hawking in their semiclassical evaluation of the wave function of the universe [21]. The key assumption is spatial homogeneity and isotropy, i.e. all degrees of freedom except the three-volume (scale factor) are frozen and the Euclidean spacetime metric is given by

$$
d s^{2}=d \tau^{2}+a^{2}(\tau) d \Omega_{3}^{2} .
$$

Here $a(\tau)$ is the scale factor depending on the proper time $\tau$ and $d \Omega_{3}^{2}$ denotes the line element on $S^{3}$. The universe topology is $S^{1} \times S^{3}$.

In the CDT model no degrees of freedom are excluded, but we integrate out all of them except the scale factor. Nevertheless, in both cases results demonstrate high similarity. The physical volume of a spatial slice for a given time $\tau$ is equal $v(\tau)=\int d \Omega_{3} \sqrt{\left.\operatorname{det} g\right|_{S^{3}}}=2 \pi^{2} a(\tau)^{3}$.

The minisuperspace action is the Einstein-Hilbert action (1) calculated for the Euclidean metric (10) [5], up to boundary terms it is given by

$$
S[a]=\frac{2 \pi^{2}}{16 \pi G} \int d \tau\left(-6 a \dot{a}^{2}-6 a+2 \Lambda a^{3}\right) .
$$

The Wick rotated Einstein-Hilbert action (1) suffers from the unboundedness of the conformal mode. As seen in the minisuperspace action (11), this is caused by the wrong sign of the kinetic term. As a consequence, the Regge action (3) is also unbounded from below but due to the UV lattice regularization it is always finite for an individual triangulation. The problem of infinities is revived when taking the continuum limit. Fortunately, in the nonperturbative approaches, like CDT, the partition function emerges as a subtle interplay of the entropic nature of triangulations and the bare action. It occurs that the entropy factor suppresses the unbounded contributions coming from the conformal factor, and the effective action for $n_{i}$ is equal to the minisuperspace action (11), but with an opposite sign of the conformal mode, and is thus bounded from below [14]. Together with a convergence of the coupling constants to their critical values, if such a point exists, the entropic and action terms should be balanced, and one hopes to obtain the proper continuum behavior.

In terms of the spatial volume, the minisuperspace action (11) can be rewritten as

$$
S[v]=-\frac{1}{24 \pi G} \int d \tau\left(\frac{\dot{v}^{2}}{v}+\beta v^{1 / 3}-3 \Lambda v\right), \quad \beta=9\left(2 \pi^{2}\right)^{2 / 3} .
$$


The classical trajectory, solving the Euler-Lagrange equation, is given by

$$
\langle v(\tau)\rangle=2 \pi^{2} R^{3} \cos ^{3}\left(\frac{\tau}{R}\right), \quad R=(\Lambda / 3)^{-1 / 2} .
$$

The physical volume $\langle v(\tau)\rangle$ describes the maximally symmetric space for a positive cosmological constant, namely the Euclidean de Sitter universe (a geometry of a four-sphere $S^{4}$ with radius $R$ ). The very same solution emerged dynamically as a background geometry in the CDT model.

\subsection{The Hausdorff dimension}

The dependence of the volume profile $\left\langle n_{i}\right\rangle$ on the total volume $N$ shows that the universe emerging in the CDT model is genuinely four dimensional. Keeping the coupling constants $K_{0}$ and $\Delta$ fixed, which naïvely means that the geometry of simplices is not changed, we measure the expectation value $\left\langle n_{i}\right\rangle$ for different total volumes $N$.

Assuming the scaling dimension is $d_{\mathrm{H}}$, the time extension expressed in lattice spacing should scale as $N^{1 / d_{\mathrm{H}}}$ and the spatial volume should scale as $N^{1-1 / d_{\mathrm{H}}}$. The physical volume, which should be independent of the number of simplices $N$, is then given by

$$
n(t) \equiv N^{-1+\frac{1}{d_{\mathrm{H}}}}\left\langle n_{i}\right\rangle, \quad t \equiv N^{-\frac{1}{d_{\mathrm{H}}}} i .
$$

For very large $N$, the scaled time variable can be treated as continuous and the normalization condition reads $\int n(t) d t=\frac{1}{N} \sum_{i} n_{i}=1$. We fit the scaling dimension $d_{\mathrm{H}}$ so that the overlap of $n(t)$ for different $N \mathrm{~s}$ is the best [2]. The best fit gives $d_{\mathrm{H}}=3.98 \pm 0.10$. The expected value $d_{\mathrm{H}}=4$ is very close to the measured result, and is well within the margin of error. Figure 6 plots the scaled three-volumes $n(t)$ for $K_{0}=2.2, \Delta=0.6$ and various total volumes $N$. It shows that $n(t)$ does not depend on $N$. From equation (9) and scaling (14) for $d_{\mathrm{H}}=4$, we get formulas

$$
n(t)=\frac{3}{4 \omega} \cos ^{3}\left(\frac{t}{\omega}\right), \quad\left\langle n_{i}\right\rangle=\frac{3 N^{3 / 4}}{4 \omega} \cos ^{3}\left(\frac{i}{\omega N^{1 / 4}}\right),
$$

where $\omega$ depends only on the coupling constants $K_{0}$ and $\Delta$, but not on $N$. For the mentioned values of the coupling constants, the measured value is $\omega \approx 0.69$. The time extent of the universe, measured in lattice units, scales as $T_{\text {univ }} \sim \omega N^{1 / 4}$. Such a scaling means that the lattice spacings behave as $a_{\mathrm{t}}, a_{\mathrm{s}} \propto N^{-1 / 4}$ which is expected for a four-dimensional spacetime.

By rescaling the continuous time $t$ to the proper time $\tau$, the scaled volume $n(t)$ can be mapped into the physical volume $v(\tau)$ given by (13) which corresponds to a four-sphere with radius $R$ [10]. 


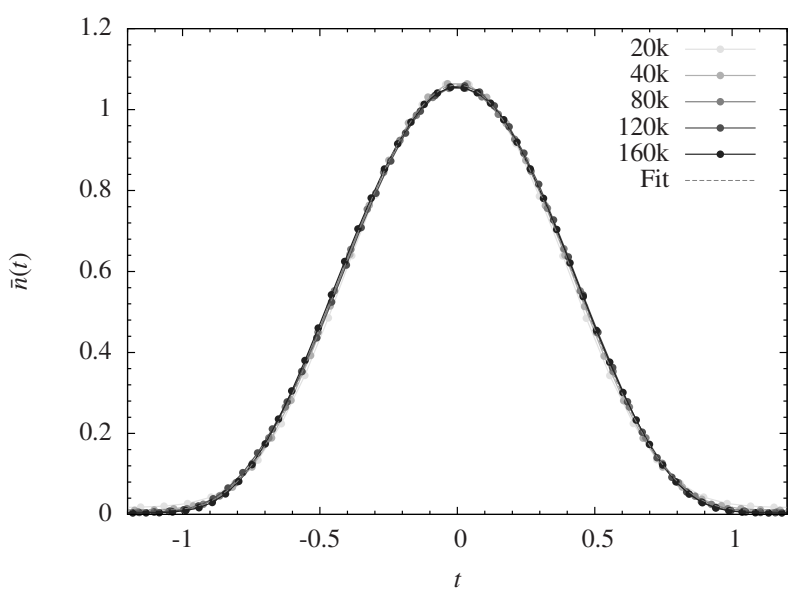

Fig. 6. Average scaled spatial volume $n(t)$ for a variety of total volumes $N$ calculated for the scaling dimension $d_{\mathrm{H}}=4$. Measured in Monte Carlo simulations for $K_{0}=2.2$ and $\Delta=0.6$. We omit the error bars not to obscure the picture. The dashed line plots the fit $n(t)=\frac{3}{4 \omega} \cos ^{3}(t / \omega)$, where $\omega=0.69$.

\subsection{The spectral dimension}

The spectral dimension $d_{\mathrm{S}}$ is another quantity revealing information about the geometry and is related to the diffusion phenomena. Let us consider such process on a $d$-dimensional manifold with a fixed and smooth Riemannian metric $g_{\mu \nu}(\boldsymbol{x})$. The probability density of finding a particle at position $\boldsymbol{x}$ after some fictitious diffusion time $\sigma$ is denoted by $\rho\left(\boldsymbol{x}, \boldsymbol{x}_{0} ; \sigma\right)$. The initial position at $\sigma=0$ is fixed at $\boldsymbol{x}_{0}, \rho\left(\boldsymbol{x}, \boldsymbol{x}_{0} ; \sigma=0\right)=\operatorname{det}^{-1 / 2} g(\boldsymbol{x}) \delta\left(\boldsymbol{x}-\boldsymbol{x}_{0}\right)$. The process is described by the diffusion equation

$$
\partial_{\sigma} \rho\left(\boldsymbol{x}, \boldsymbol{x}_{0} ; \sigma\right)=\triangle_{g} \rho\left(\boldsymbol{x}, \boldsymbol{x}_{0} ; \sigma\right),
$$

where $\triangle_{g}$ is the Laplace operator corresponding to $g_{\mu \nu}(\boldsymbol{x})$. The return probability of finding a particle at the initial point after diffusion time $\sigma$ can be used to calculate the spectral dimension $d_{\mathrm{S}}$ defined by

$$
d_{\mathrm{S}} \equiv-2 \frac{d \log P(\sigma)}{d \log \sigma} .
$$

The average return probability $P(\sigma)$ in (17), supplying a global information about geometries, is given by

$$
P(\sigma)=\left\langle\frac{1}{V_{4}} \int d^{d} \boldsymbol{x} \sqrt{\operatorname{det} g(\boldsymbol{x})} \rho(\boldsymbol{x}, \boldsymbol{x} ; \sigma)\right\rangle,
$$


where $V_{4}=\int d^{d} \boldsymbol{x} \sqrt{\operatorname{det} g(\boldsymbol{x})}$ is the total spacetime volume and the average is also performed over the ensemble of geometries. For Euclidean flat manifold $\mathbb{R}^{d}$, the spectral dimension $d_{\mathrm{S}}$ calculated from (17) and (18) is equal to the topological dimension $d$ and Hausdorff dimensions $d_{\mathrm{H}}$.

Since it is easy to perform numerical simulations of a diffusion process in the CDT framework, the measurement of the return probability and the spectral dimension is particularly convenient. While the spacetime geometry is regularized by piecewise flat manifolds built from foursimplices, the diffusion equation (16) is given by its discrete counterpart $\rho\left(i, i_{0} ; \sigma+1\right)=\frac{1}{5} \sum_{j \leftrightarrow i} \rho\left(j, i_{0} ; \sigma\right)$. Here, the sum is evaluated over all simplices $j$ adjacent to $i$. To evaluate $\rho\left(i, i_{0} ; \sigma\right)$, we pick an initial four-simplex $i_{0}$ lying in the central slice, impose the initial condition $\rho\left(i, i_{0} ; \sigma=0\right)=\delta_{i i_{0}}$ and iterate the diffusion equation. We repeat the above operations for a number of random starting points $i_{0}(\sim 100)$ and a number of configurations $(\sim 1000)$. Further, we calculate the average return probability $P(\sigma)$, and the spectral dimension $d_{\mathrm{S}}$ from $(17)$.

Figure 7 shows the measured spectral dimension $d_{\mathrm{S}}$ as a function of the diffusion time steps $\sigma$. For small values of $\sigma<30$, because of strong lattice artifacts, the spectral dimension cleaves for even and odd time steps. As a result of the finite volumes of configurations, for very large $\sigma \gg 500$, the spectral dimension $d_{\mathrm{S}}$ eventually falls down to zero. In the presented range $40<\sigma<500$, the spectral dimension can be expressed by the formula

$$
d_{\mathrm{S}}(\sigma)=a-\frac{b}{c+\sigma}=4.02-\frac{120}{58+\sigma},
$$

where variables $a, b$ and $c$ were obtained from the best fit. Because the spectral dimension changes in diffusion time, it is scale dependent. Small $\sigma$, means that the diffusion process probes only the nearest vicinity of the initial point. Extrapolation of results gives the short-distance limit of the spectral dimension

$$
d_{\mathrm{S}}(\sigma \rightarrow 0)=1.95 \pm 0.10 \text {. }
$$

In the long-distance limit, the spectral dimension tends to

$$
d_{\mathrm{S}}(\sigma \rightarrow \infty)=4.02 \pm 0.05
$$

The short-range value of the spectral dimension $d_{\mathrm{S}}=2$, much smaller than the scaling dimension $d_{\mathrm{H}}$, suggests a fractal nature of geometries appearing in the path integral at short distances. At long distances $d_{\mathrm{S}}=4$, and configurations resemble a smooth manifold. Amazingly, such nontrivial scale dependence of the spectral dimension of the quantum spacetime, the same infrared $\left(d_{\mathrm{S}}=4\right)$ and ultra-violet $\left(d_{\mathrm{S}}=2\right)$ behavior, is also present in HořavaLifshitz gravity [15] and in the Renormalization Group approach [16]. 


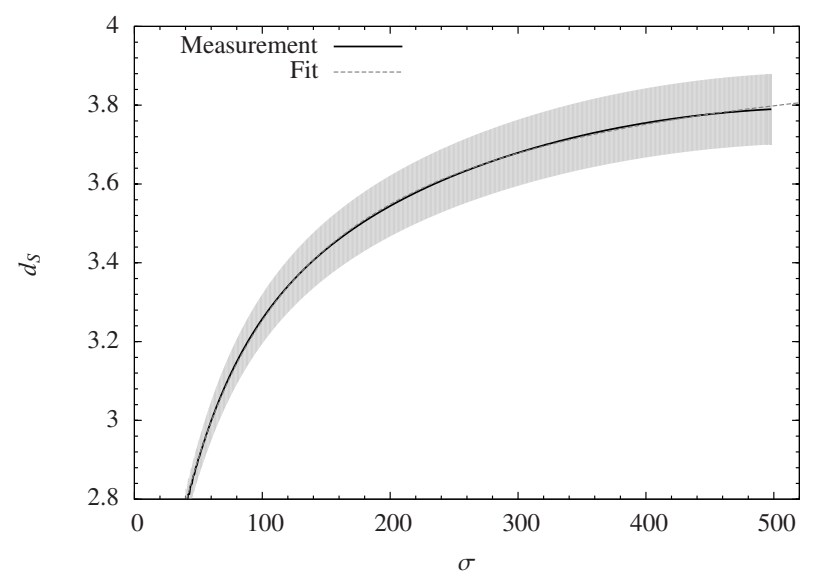

Fig. 7. The spectral dimension $d_{\mathrm{S}}$ of the universe as a function of diffusion time $\sigma$, measured for $K_{0}=2.2, \Delta=0.6$ and $N=160 \mathrm{k}$. The thick curve plots the average measured spectral dimension, while the highlighted area represents the error bars. The best fit $d_{\mathrm{S}}(\sigma)=4.02-\frac{120}{58+\sigma}$ is drawn with a dashed line.

\section{The effective action}

The minisuperspace model describes with great accuracy the background geometry which emerged in the CDT model. This encourages us to check whether it also properly describes quantum fluctuations around the classical trajectory. The model of Causal Dynamical Triangulations is a genuinely nonperturbative approach to quantum gravity. It takes into account both the entropy factor and the bare action (3). However, we can consider the semiclassical limit of the lattice approach and extract the effective action that controls the three-volume $n_{i}$. In analogy to the path integral formulation of quantum mechanics, $n_{i}$ describes the position at discrete time $i$ of a nonphysical particle trajectory, giving a contribution to the partition function. Let us denote the deviation of $n_{i}$ from the expectation value $\left\langle n_{i}\right\rangle$ by

$$
\eta_{i}=n_{i}-\left\langle n_{i}\right\rangle .
$$

In the semiclassical approximation, we expand the effective action up to quadratic terms in $\eta_{i}$

$$
S[n=\langle n\rangle+\eta] \approx S[\langle n\rangle]+\frac{1}{2} \sum_{i, j} \eta_{i} \boldsymbol{P}_{i j} \eta_{j}+O\left(\eta^{3}\right) .
$$

The sum is performed over time slices $i, j=1 \ldots T$. The Sturm-Liouville operator $\boldsymbol{P}$ carries information about quantum fluctuations and may be extracted from numerical data. 
Similarly to $\left\langle n_{i}\right\rangle$, we measure the covariance matrix $\boldsymbol{C}$ of volume fluctuations using Monte Carlo techniques (7)

$$
\boldsymbol{C}_{i j} \equiv\left\langle\eta_{i} \eta_{j}\right\rangle
$$

If the quadratic approximation is accurate, the propagator $C$ and the matrix $\boldsymbol{P}$ are directly related, $\boldsymbol{C}=\boldsymbol{P}^{-1}$.

For numerical convenience the measurements were performed only for triangulations with a fixed total volume $N$. Such constraint introduces a zero eigenvalue to the spectrum of $\boldsymbol{C}$. Because a straightforward inversion is not possible, we first project $\boldsymbol{C}$ on the subspace orthogonal to the corresponding eigenvector. Details of this procedure are described in [12]. We measure in Monte Carlo simulations the covariance matrix $\boldsymbol{C}$, and subsequently extract $\boldsymbol{P}$ which can then be compared with the predictions of the minisuperspace model.

It was shown that the empirical effective action indeed corresponds to a discretization of the minisuperspace action (12) up to an overall sign [17]. A discretized, dimensionless version of the action (12) is given by

$$
S[n]=\sum_{i} g_{1} \frac{\left(n_{i+1}-n_{i}\right)^{2}}{n_{i+1}+n_{i}}+g_{2} n_{i}^{1 / 3}-g_{3} n_{i} .
$$

The constants $G, \Lambda$ and $\beta$ present in (12) are incorporated into $g$ s. We used the standard discretization of the time derivative, $\dot{v} \rightarrow n_{i+1}-n_{i}$. Inside the blob region, the discrete volume $n_{i}$ may be treated as a continuous variable. Because the stalk region is governed by very strong lattice artifacts, the semiclassical approximation is not reliable there.

The Sturm-Liouville operator defined in (20) can be calculated from

$$
\boldsymbol{P}_{i j}=\left.\frac{\partial^{2} S[n]}{\partial n_{i} \partial n_{j}}\right|_{n=\langle n\rangle} .
$$

For action (22), it is given by

$$
\eta_{i} \boldsymbol{P}_{i j} \eta_{j}=\frac{\left(\eta_{i+1}-\eta_{i}\right)^{2}}{k_{i}}-u_{i} \eta_{i}^{2}, \quad k_{i}=g_{1}^{-1}\left\langle n_{i}\right\rangle, \quad u_{i}=g_{2}\left\langle n_{i}\right\rangle^{-5 / 3}
$$

The kinetic part $k_{i}$ is proportional to the average volume $\left\langle n_{i}\right\rangle$ and the potential part $u_{i}$ behaves like $\left\langle n_{i}\right\rangle^{-5 / 3}$.

As expected, up to a numerical noise, the measured matrix $\boldsymbol{P}$ has a tridiagonal structure and the coefficients $\left\{k_{i}\right\}$ and $\left\{u_{i}\right\}$ can be extracted from empirical data. The nonzero subdiagonal elements of $\boldsymbol{P}$ come from the 
kinetic part, which couples volumes of successive slices. Additionally, the potential part contributes to the diagonal. Indeed, the measured values agree with the expectations for the minisuperspace model (23). Figure 8 presents the measured values of $g_{1} k_{i}$ and $\left\langle n_{i}\right\rangle$ for various total volumes $N$. The theoretical fit (23) agrees very well with the measured quantities. Additionally, the effective coupling constant $g_{1}$ does not depend on $N$ in the margin of error. For $K_{0}=2.2, \Delta=0.6$, we measured $g_{1}=0.038 \pm 0.002$. The kinetic part of the quantum fluctuations is described by the minisuperspace action (12).

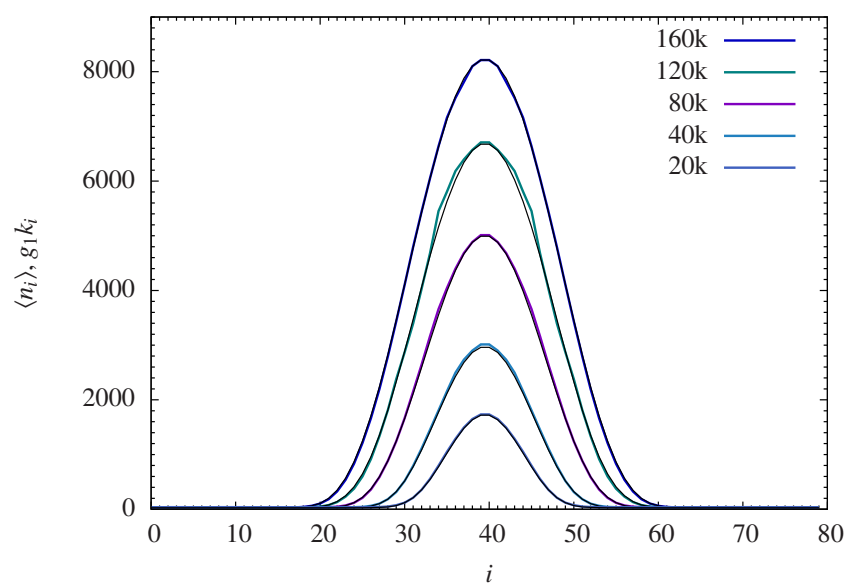

Fig. 8. Kinetic term: The directly measured expectation values $\left\langle n_{i}\right\rangle$ (black line), compared to $g_{1} k_{i}$ (thick gray line) extracted from the measured covariance matrix $C$ for $K_{0}=2.2, \Delta=0.6$ and various total volumes $N$ ranging from 20000 to 160000 simplices. The theoretical prediction $g_{1} k_{i}=\left\langle n_{i}\right\rangle$ is realized with a very high accuracy. The value of $g_{1}$ is constant for all volumes $N$.

The same is true for the potential term $\left\{u_{i}\right\}$. Figure 9 shows the measured values of coefficients $u_{i}$ extracted from the empirical matrix $\boldsymbol{P}$ as a function of $\left\langle n_{i}\right\rangle$. Because the kinetic term is dominating in the continuum limit $N \rightarrow \infty$, in this case the statistical errors are larger. The physically interesting region of large volumes corresponds to relatively small values of $u_{i}$ as they are expected to fall as $\left\langle n_{i}\right\rangle^{-5 / 3}$. Nonetheless, due to sufficiently long Monte Carlo sample, the results confirm that the formula (23) is in agreement with measurements. The selected range of $n_{i}$ corresponds to the bulk. The best fit of the form $f(x)=a x^{-c}$ to the empirical values $u_{i}$ as a function of $\left\langle n_{i}\right\rangle$ gives $c=-1.658 \pm 0.096$ which is very close to the theoretical value $c=-5 / 3$. The fit $f(x)=a x^{-5 / 3}$, corresponding to the potential part of action (22) is presented in Fig. 9 with a thin line. 


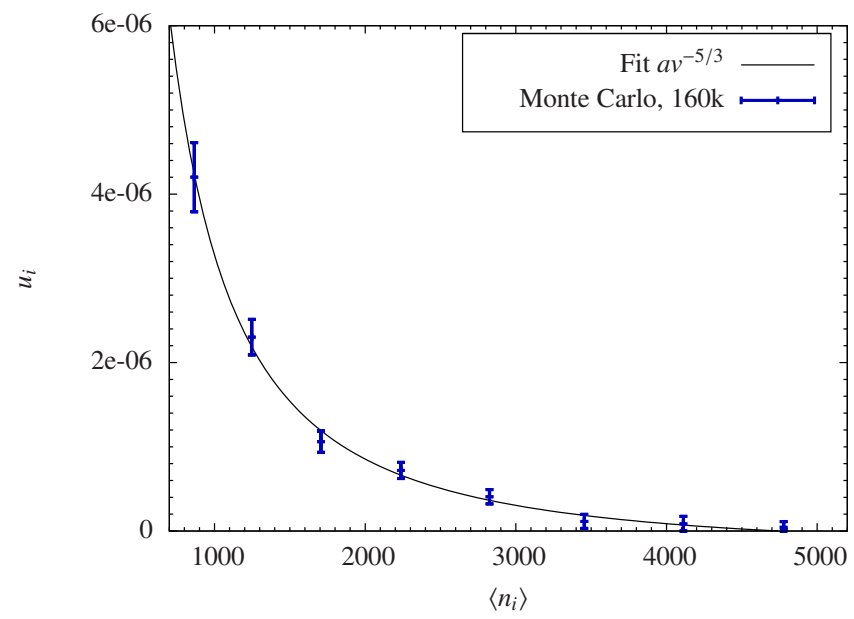

Fig. 9. The extracted potential term $u_{i}$ as a function of average volume $\left\langle n_{i}\right\rangle$. The fit $c_{2} \bar{v}_{t}^{-5 / 3}$ presents the behavior expected for the minisuperspace model. The visible points correspond to the blob region.

Both the kinetic part as well as the potential part of the measured SturmLiouville operator $\boldsymbol{P}$ agree with the expectations for the minisuperspace action. Recently, the effective action was determined using a new, more direct, method based on the transfer matrices [22]. Those results are consistent with the one presented in this article.

\section{Conclusions}

The model of Causal Dynamical Triangulations is a nonperturbative and background independent approach to quantum gravity. The foundation of this model is the formalism of path-integrals applied to quantize a theory of gravitation. This method is a natural generalization of discretization procedure, introduced in the definition of quantum mechanical Feynman's path integral, to higher dimensions. The introduction of Wick rotation allows us to use very powerful Monte Carlo techniques and calculate quantum expectation values of observables.

Among the three phases, the de Sitter phase seems to be physically relevant. In this phase, the time translational symmetry is spontaneously broken and the scale factor as a function of time is given by a bell-shaped distribution. Recent results show that a universe emerges dynamically in Causal Dynamical Triangulations. Its geometry is genuinely four-dimensional and corresponds to de Siter space, the maximally symmetric solution to the classical Einstein equations in the presence of a positive cosmological constant. At large scales, both the Hausdorff and spectral dimensions are equal 4. 
CDT presents a picture of the universe with superimposed finite quantum fluctuations around the classical trajectory. The measurements of the covariance matrix in the semiclassical regime allowed us to reconstruct the discrete effective action describing quantum fluctuations of the three-volume $n_{i}$. The effective action can be identified with the minisuperspace action, although the CDT model does not reduce any degrees of freedom.

The author would like to thank Jerzy Jurkiewicz and Jan Ambjørn for introducing him into this fascinating topic and for a fruitful collaboration. The author acknowledges support from the ERC-Advance grant 291092, "Exploring the Quantum Universe" (EQU).

\section{REFERENCES}

[1] J. Ambjørn, J. Jurkiewicz, R. Loll, Phys. Rev. Lett. 85, 924 (2000) [arXiv:hep-th/0002050].

[2] J. Ambjørn, J. Jurkiewicz, R. Loll, Phys. Rev. D72, 064014 (2005) [arXiv:hep-th/0505154].

[3] J. Ambjørn, J. Jurkiewicz, R. Loll, Nucl. Phys. B610, 347 (2001) [arXiv:hep-th/0105267].

[4] R.M. Wald, General Relativity, The University of Chicago Press, 1984.

[5] G.W. Gibbons, S.W. Hawking, Phys. Rev. D15, 2752 (1977).

[6] C. Teitelboim, Phys. Rev. Lett. 50, 705 (1983).

[7] C. Teitelboim, Phys. Rev. D28, 297 (1983).

[8] J. Ambjørn, J. Jurkiewicz, Phys. Lett. B278, 42 (1992).

[9] J. Ambjørn, A. Görlich, S. Jordan, J. Jurkiewicz, R. Loll, Phys. Lett. B690, 413 (2010) [arXiv: 1002.3298 [hep-th]].

[10] A. Görlich, Lect. Notes Phys. 863, 93 (2013).

[11] A. Görlich, Acta Phys. Pol. B 39, 3343 (2008).

[12] J. Ambjørn, A. Görlich, J. Jurkiewicz, R. Loll, Phys. Rev. D78, 063544 (2008) [arXiv:0807.4481 [hep-th]].

[13] J. Ambjørn, A. Görlich, J. Jurkiewicz, R. Loll, Phys. Rep. 519, 127 (2012).

[14] A. Dasgupta, R. Loll, Nucl. Phys. B606, 357 (2001) [arXiv:hep-th/0103186].

[15] P. Hořava, Phys. Rev. Lett. 102, 161301 (2009) [arXiv:0902.3657 [hep-th]].

[16] O. Lauscher, M. Reuter, J. High Energy Phys. 0510, 050 (2005) [arXiv:hep-th/0508202].

[17] J. Ambjørn, A. Görlich, J. Jurkiewicz, R. Loll, Phys. Rev. Lett. 100, 091304 (2008) [arXiv:0712.2485 [hep-th]]. 
[18] J. Ambjørn, A. Görlich, J. Jurkiewicz, R. Loll, Acta Phys. Pol. B 39, 3309 (2008).

[19] J. Ambjørn, A. Görlich, J. Jurkiewicz, R. Loll, Phys. Lett. B690, 420 (2010) [arXiv:1001.4581 [hep-th]].

[20] J. Ambjørn et al., Nucl. Phys. B849, 144 (2011).

[21] J.B. Hartle, S.W. Hawking, Phys. Rev. D28, 2960 (1983).

[22] J. Ambjørn, J. Gizbert-Studnicki, A. Görlich, J. Jurkiewicz, J. High Energy Phys. 1209, 017 (2012). 